Research Article

\title{
Measuring and Modelling Nonlinear Elasticity of Ex Vivo Mouse Muscles
}

\author{
E. Rizzuto $\left(\mathbb{D},{ }^{1}\right.$ R. De Luca, ${ }^{1}$ A. Musarò, ${ }^{2}$ and Z. Del Prete ${ }^{1}$ \\ ${ }^{1}$ Department of Mechanical and Aerospace Engineering, Sapienza University of Rome, Rome, Italy \\ ${ }^{2}$ Laboratory Affiliated to Istituto Pasteur Italia-Fondazione Cenci Bolognetti, \\ DAHFMO-Unit of Histology and Medical Embryology, Sapienza University of Rome, Rome, Italy
}

Correspondence should be addressed to E. Rizzuto; emanuele.rizzuto@uniroma1.it

Received 1 March 2021; Accepted 28 October 2021; Published 17 November 2021

Academic Editor: Emiliano Schena

Copyright (c) 2021 E. Rizzuto et al. This is an open access article distributed under the Creative Commons Attribution License, which permits unrestricted use, distribution, and reproduction in any medium, provided the original work is properly cited.

Elastography is a noninvasive imaging technique that provides information on soft tissue stiffness. Young's modulus is typically used to characterize soft tissues' response to the applied force, as soft tissues are often considered linear elastic, isotropic, and quasi-incompressible materials. This approximation is reasonable for small strains, but soft tissues undergo large deformations also for small values of force and exhibit nonlinear elastic behavior. Outside the linear regime, the elastic modulus is dependent on the strain level and is different for any kind of tissue. The aim of this study was to characterize, ex vivo, the mechanical response of two different mice muscles to an external force. A system for transverse force-controlled uniaxial compression enabled obtaining the stress-strain $(\sigma-\varepsilon)$ curve of the samples. The strain-dependent Young's modulus (SYM) model was adopted to reproduce muscle compression behavior and to predict the elastic modulus for large deformations. After that, a recursive linear model was employed to identify the initial linear region of the $\sigma-\varepsilon$ curve. Results showed that both muscle types exhibited a strain hardening effect and that the SYM model provided good fitting of the entire $\sigma-\varepsilon$ curves. The application of the recursive linear model allowed capturing the initial linear region in which the approximation of these tissues as linear elastic materials is reasonable. The residual analysis displayed that even if the SYM model better summarizes the muscle behavior on the entire region, the linear model is more precise when considering only the initial part of the $\sigma-\varepsilon$ curve.

\section{Introduction}

Noninvasive measurement of soft biological tissues' mechanical properties is of significant clinical interest as pathologies are generally correlated with changes in tissue stiffness. Many cancers, such as breast and prostate ones, typically appear stiffer than the surrounding tissues, and diffuse diseases, such as cirrhosis of the liver, are known to significantly increase the stiffness of the liver tissue as a whole [1-5]. In addition to pathology, there is evidence that consistent elastic contrast also exists among various normal tissues $[1,6,7]$. Imaging these differences in soft tissue stiffness is commonly obtained using elastography $[2,3,8-11]$, which provides noninvasive images of tissue mechanical characteristics, at depth, with high resolution and good contrast.
To date, there are several elastography imaging techniques which are based on the same principle: an external force is applied to the tissue, and the local internal displacement is measured by either ultrasound or magnetic resonance imaging to be converted in a suitable parameter to display (e.g., strain, Young's modulus, and shear wave speed). The external force may be applied in different ways: a dynamic force is mandatory to generate a shear wave and thus to measure and image its speed, while the applied force may be dynamic, quasistatic, or static if there is need to image displacement or strain $[2,3]$. The importance of measuring tissue mechanical properties lies in the fact that they may be considered diagnostic indicators which provide additional and clinically relevant information for the characterization of soft tissues and for the discrimination between normal and abnormal tissues. In this context, a wide 
experience with transient and shear wave elastography to assess diffuse liver diseases exists [3, 9, 12]. Transient elastography allows for a reduction of more than $50 \%$ of liver biopsies which, to date, still represents the gold standard technique for the diagnosis of liver chronic disease, even if biopsies are invasive and painful and may cause anxiety to the patients $[13,14]$. Strain and shear wave elastography are typically used to examine breast, thyroid, gastrointestinal tract, prostate, and musculoskeletal system [9, 15-18]. For instance, shear wave elastography improves the specificity of breast ultrasound imaging, has high reproducibility for breast masses [19-22], and provides information of the functional changes accompanying musculoskeletal pathologies [23-25].

Soft tissues are viscoelastic, poroelastic, anisotropic, and nonlinear materials. In practice, many assumptions are made to display their mechanical properties using elastography: they are typically modelled as linear elastic, isotropic, and quasi-incompressible materials [6]. Under these conditions, one single parameter is needed to characterize the response of a tissue to the applied mechanical force: Young's modulus $[6,26]$. However, when scanning with the ultrasound probe, a precompression is applied by the operator, and soft tissues, being nonlinear, exhibit a strain hardening effect; that is, they get stiffer as the amount of applied pressure increases [27, 28]. For large deformations, they show a nonlinear elastic behavior as their modulus is dependent on the strain level, and the increase in the modulus varies from one tissue type to another $[6,29,30]$. In strain elastography, large strains may be required to achieve a reasonable strain signal-to-noise ratio and to enhance the contrast between healthy and pathological tissues [30]. Nevertheless, strains outside the linear elastic region do not provide reliable information about tissue material properties, since Young's modulus for high strain is largely stressdependent [26]. High strain elasticity measurements provide information only about tissue pseudoelastic properties, that is, elasticity of tissue at a specific stress or strain level. In shear wave elastography, the probe must be applied very lightly with a generous amount of gel [25, 31]; otherwise, abnormal stiffness is shown; for instance, in breast imaging, fat tissue may be measured to have the same elasticity pattern of cancer if a high precompression is applied [29]. A proper assessment of the force applied during elastography techniques may be therefore used, together with the proper mathematical model, as a real-time correction of the measurements to avoid misinterpretation of the results.

Within this context, the present work aims at investigating and modelling the elastic behavior of two different mice muscles. A mechanical system able to apply an external force and to simultaneously measure the subsequent displacement of the sample is used to obtain, ex vivo, the stressstrain $(\sigma-\varepsilon)$ curves of Tibialis Anterior (TA) and Extensor Digitorum Longus (EDL) muscles. These curves are then employed to find the mathematical models that describe the elastic behavior of the investigated soft tissues and to identify the limits of the linear stress-strain regions, as well as to reconstruct the Young's modulus values corresponding to high strain levels.

\section{Theory}

The elasticity of a solid material describes its tendency to return to the original shape and size after being subjected to a deforming force. Soft biological tissues are nonlinear, viscoelastic, anisotropic, and nonhomogeneous materials, and their properties are dependent on time. Moreover, the resistance to deformation increases when the applied stress increases [32], so that multiple values of Young's modulus could be obtained, as it depends on the applied stress $[26,32]$. In general, it is assumed that soft biological tissues behave as linear elastic, isotropic materials [32] but, in vivo, soft tissues usually undergo large deformations and these assumptions become invalid [33]. To characterize the nonlinear elastic response of soft tissues and to overcome the difficulty in obtaining Young's modulus by force-deformation test, several theoretical models have been formulated $[33,34]$. In particular, Li et al. [35] proposed a strain-dependent Young's modulus (SYM) to introduce nonlinearity in the general theory of elasticity for transversely isotropic bodies, as reported in the following equation:

$$
\begin{aligned}
& E_{L}=k_{1} e^{k_{2} \varepsilon}, \\
& E_{T}=k_{3} e^{k_{4} \varepsilon},
\end{aligned}
$$

where $E_{L}$ and $E_{T}$ are the longitudinal and transverse Young's moduli, respectively, and $k_{1}, k_{2}, k_{3}$, and $k_{4}$ are the model parameters. The authors applied this model to reproduce the tensile behavior of porcine aortic heart valves, whereas Van Loocke and coauthors $[33,36]$ adapted it to reproduce skeletal muscle tissue compression behavior. In our study, the SYM model was used to investigate the passive elastic behavior of ex vivo mouse skeletal muscles, and model parameters are determined by fitting the experimental data $(\varepsilon, \sigma)$.

\section{Experimental Setup and Protocol}

3.1. Animals. Six C57BL/6 female mice (15-17 week old), bred at the DAHFMO-Unit of Histology and Medical Embryology Laboratories at Sapienza University of Rome, were used. All the experiments were conducted within the animal welfare regulations and guidelines of the Italian national law D.L. 04/03/2014, n.26, about the use of animals for research. From each animal, one Tibialis Anterior (TA) muscle and one Extensor Digitorum Longus (EDL) muscle were excised and subjected to uniaxial unconfined compression in the cross-fiber. After the dissection, the samples were kept soaked in a physiological solution at room temperature and the measurements were completed within 30 minutes. As the test typically lasted few minutes, dehydration of the sample was not an issue. No preconditioning was applied to the samples and each sample was tested once to avoid permanent deformation due to the high strain level induced [36]. A preload ranging from $6 \mathrm{mN}$ to $9 \mathrm{mN}$ was applied to the sample to attach it to the indentor. 
3.2. Uniaxial Unconfined Compression Test. Two methods are typically employed to assess the mechanical characteristics of soft biological tissues: probe indentation and tensile stretching. Even though both techniques provide a description of the material response to an applied stress, they are distinct. Strictly speaking, the adopted method of deformation must be considered to make empirical values of Young's modulus reliable, consistent, and nonambiguous [32]. In this study, a force-controlled uniaxial compression test has been conducted on fresh mouse tissues ex vivo [37]. The experimental system was based on a dual mode actuator/transducer 305C-LR (Aurora Scientific Inc., Aurora, Canada) able to measure and control both length and force, with length signal resolution of $1 \mu \mathrm{m}$ and force signal resolution of $1 \mathrm{mN}$. The motor was controlled through a DAQ NI-PCIe-6363X (National Instruments, Austin, TX, USA), and custom-made software developed in LabView 2012 (National Instruments, Austin, TX, USA) allowed setting the shape and the intensity of the applied loading profile. A circular indentor (Aurora Scientific Inc., Aurora, Canada) of $3 \mathrm{~mm}$ of diameter was rigidly attached to the lever arm of the actuator/transducer. The sample was placed in touch with the piston with the muscle fibers perpendicular to the direction of compression and remained unconstrained laterally. The motor was controlled in force and the specimen was subjected to a compressive ramp whose maximum force was $200 \mathrm{mN}$ at $50 \mathrm{~s}$. The loading speed was set at $4 \mathrm{mN} / \mathrm{s}$ and was chosen reasonably low to limit the viscosity effects. The applied signals were force-controlled to mimic the use of a scanning probe in elastography applications, and the true applied force and displacement were recorded for offline processing. All the instrumentations were placed on an isolation workstation with Faraday cage (Newport Vision IsoStation, California, USA).

3.3. Recursive Linear Fitting Model. Even though soft biological tissues exhibit a nonlinear $\sigma-\varepsilon$ behavior, they are typically assumed to be linear elastic if a significant linear region of the $\sigma-\varepsilon$ curve exists in the limit of small deformation to the applied stress [32], and elastography is generally based on this approximation $[3,6,26]$. Here, a recursive linear fitting model was developed to calculate the strain range in which each tested sample could be reasonably considered a linear elastic solid. This model yielded to obtain the best linear fitting curve in terms of $R^{2}$ value, thus allowing to assess the highest deformation $\left(\varepsilon_{\max }\right)$ of the linear regime. Young's modulus of the linear region $\left(E_{\mathrm{lin}}\right)$ was then obtained as the slope of this curve [37].

\subsection{Optical System to Measure Sample Characteristic} Dimension. Measurement of the sample geometrical dimensions was made using an optical methodology. This system consisted of an ACA2040-180 km monochromatic camera (Basler, Ahrensburg, Germany) mounted on a SMZ800N (Nikon Inc., Melville, NY, USA) stereomicroscopy equipped with a 0.5 Plan Apo objective (Nikon) to capture the whole longitudinal length of the specimen. This camera had a pixel size of $5.5 \mu \mathrm{m} \times 5.5 \mu \mathrm{m}$ and a full resolution of $2048 \times 2048$ pixels. The lighting was provided by a PL-3000 cold light illuminator (Photonic Optics, Vienna, Austria) that guaranteed a light field intensity up to $26 \mathrm{Mlx}$, and a NI-PCIe 1433 frame grabber (National Instruments, Austin, TX, USA) was employed for image acquisition. A $45^{\circ}$ angled mirror (Nikon) was used to image the transverse length $w_{0}$ of the sample (Figure 1).

For each test, an image prior compression was acquired to assess the number of pixels $\left(N_{p}\right)$ in the length $(h)$ of the circular piston (Figure 1(a)), which was used as calibrating target. Being $h=1.00 \mathrm{~mm}$ (this measurement was made using an analog caliper with an accuracy of $0.05 \mathrm{~mm}$ ), the pixel size was obtained dividing $h$ by $N_{p}$. This value was then used to calculate the width $\left(w_{0}\right)$ of the specimen when the preload was applied (Figure 1(b)). All the pixel assessments were done by using ImageJ software (National Institutes of Health, Bethesda, Maryland, USA). The average characteristic dimension $w_{0}$ of the specimens was $2.00 \pm 0.28 \mathrm{~mm}$ and $0.89 \pm 0.11 \mathrm{~mm}$ for the TA and EDL groups, respectively.

3.5. Statistical Analysis. Differences in the measured maximum strain, in the maximum value of Young's modulus computed with the SYM model, in the maximum strain value computed with the linear model, and in the maximum value of Young's modulus computed with the linear model between EDL and TA groups were evaluated with unpaired $t$-test, and the difference was considered significant for $p$ value $<0.05$. Differences in the residual distributions between the linear model and the SYM model were evaluated with Man$\mathrm{n}$-Whitney rank-sum test, and the difference in the median values between the two groups was considered significant for $p$ value $<0.05$. Statistical analysis was performed with GraphPad Prism 6.0 (GraphPad Software, Inc).

\section{Results and Discussion}

Figure 2 shows the mean and standard deviation of the strain-stress relationship measured from the applied force and the consequent displacement of 5 TA muscles and 6 EDL muscles. As expected, for both muscle types, the strain increased nonlinearly as a function of the applied stress, and each muscle type exhibited a different elastic behavior. In particular, the average maximum strain value was $43 \%$ higher $(p<0.01)$ in the EDL group than in the TA group.

To quantify the strain hardening effect of the investigated mouse muscles, the exponential fitting proposed by Li et al. [35] was exploited. The adopted model provided a very good fit to the experimental data, as shown in Table 1, and allowed reconstructing the values of Young's modulus for each level of strain undertaken by each specimen.

Figure 3(a) shows an example of the experimental results fitted with the exponential model for a TA sample, and Figure 3(b) displays the corresponding reconstructed Young's modulus as a function of strain. Of note, the average maximum Young's modulus obtained from the SYM model was significantly $(p<0.01)$ higher in the TA muscles than in the EDL muscles. 


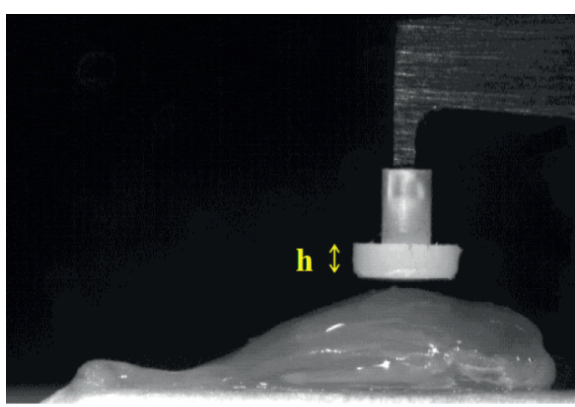

(a)

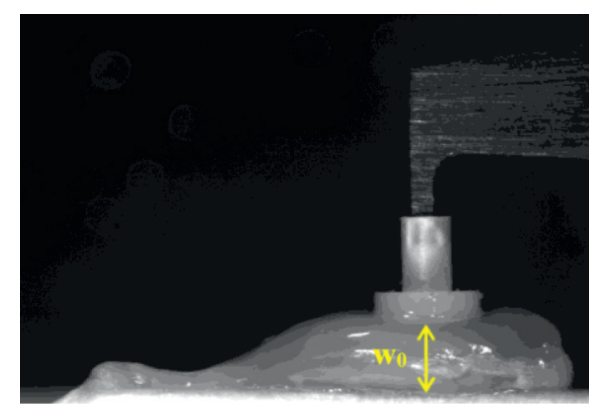

(b)

FIgURE 1: Example of images of a TA muscle acquired by the optical system. The indentor and the lever arm of the actuator/transducer were also captured. (a) Precompression image: $h$ is the length of the circular piston. (b) Image acquired after a preload of $8 \mathrm{mN}$ was applied: $w_{0}$ is the initial length of the sample in the cross-fiber direction.

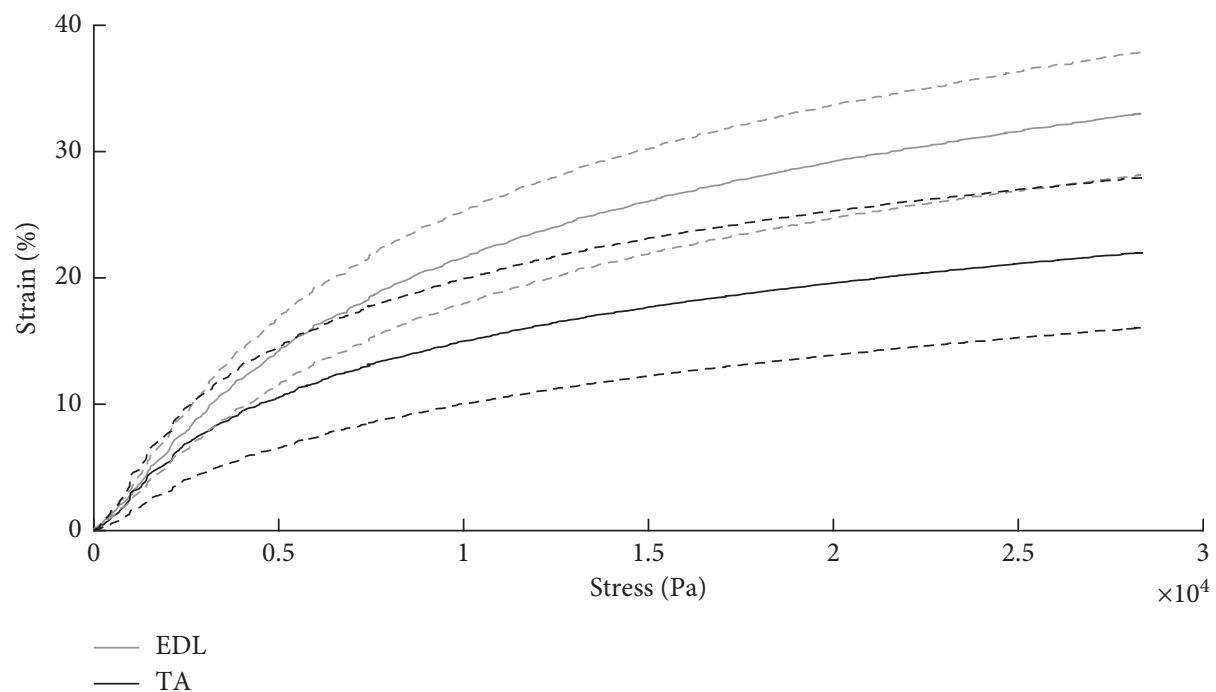

Figure 2: Mean (solid line) and standard deviation (dotted line) of the experimental strain-stress curve for the TA (grey) and EDL (black) groups of samples. Average data are expressed as mean \pm SD. $n: 5$ TA and 6 EDL.

TABLE 1: $R^{2}$ values of exponential models and the corresponding maximum value of the reconstructed Young's modulus for TA and EDL specimens.

\begin{tabular}{lcccc}
\hline \multirow{2}{*}{ Mouse } & \multicolumn{2}{c}{ TA } & \multicolumn{2}{c}{ EDL } \\
& $R^{2}$ & $E_{\max }(\mathrm{kPa})$ & $R^{2}$ & $E_{\max }(\mathrm{kPa})$ \\
\hline 1 & 0.9992 & 138.65 & 0.9984 & 75.33 \\
2 & 0.9996 & 130.34 & 0.9976 & 87.98 \\
3 & 0.9994 & 121.69 & 0.9963 & 80.59 \\
4 & 0.9994 & 118.62 & 0.9989 & 105.64 \\
5 & 0.9974 & 101.61 & 0.9973 & 70.58 \\
6 & - & - & 0.9987 & 97.06 \\
Average & & $122.18 \pm 13.91^{* *}$ & & $86.20 \pm 13.36$ \\
\hline
\end{tabular}

Data reported here refer to the whole loading profile. Average data are expressed as mean \pm SD. ${ }^{* *} p<0.01$ versus EDL.

Table 2 displays reconstructed $E_{\text {lin }}$ and $\varepsilon_{\max }$ obtained through the recursive linear fitting for both muscle types. Results showed that the initial region of the $\sigma-\varepsilon$ curve is highly linear $(R>0.98)$ for strains up to $8.18 \pm 1.51 \%$, on average, for TA muscles, and to $13.26 \pm 2.29 \%$, on average, for EDL muscles, with this difference being statistically significant $(p<0.01)$. As a result of this, Young's modulus associated with the entire linear region was found to be $38.12 \pm 13.91 \mathrm{kPa}$, on average, for TA muscles, and $33.46 \pm 6.5 \mathrm{kPa}$, on average, for EDL muscles. The higher variability obtained for the linear model is, in our opinion, related to the fact that this value is computed on the very first part of the stress-strain curve, the one with the higher slope. Any small differences in the maximum strain determined on the basis of our recursive algorithm may therefore lead to high differences in the $E$ values. Nonetheless, this variability obtained in the estimated modulus even for samples that are excised from very similar and controlled animal confirms the need for assessing the exact elasticity for each sample of interest.

Figure 4 depicts an example of the two fitting models for one EDL specimen, highlighting that, for small strains, the linear model provides better fitting than the exponential one.

To evaluate the goodness of fitting, we performed a residual analysis for the two models: for each specimen, we focused on the initial linear region of the $\sigma-\varepsilon$ curve previously computed and calculated the difference between the measured value and the value reconstructed with the linear model 


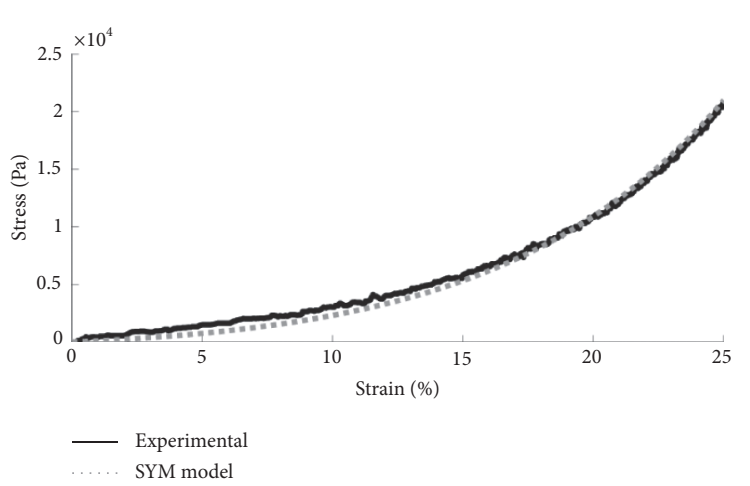

(a)

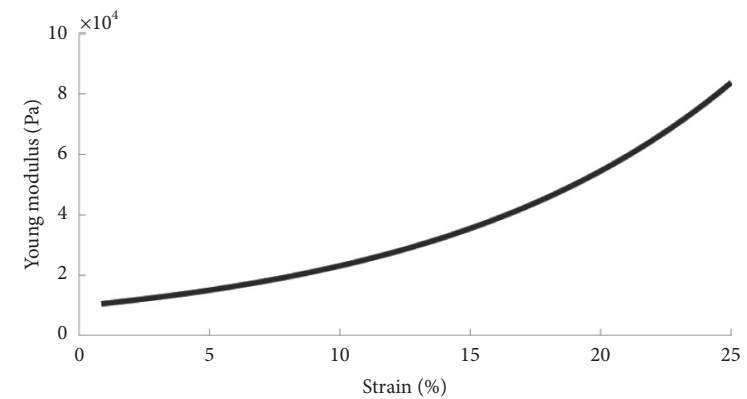

(b)

FIGURE 3: (a) Experimental stress-strain curve of one TA specimen (solid) and the corresponding exponential fitting model curve (dotted). (b) Reconstructed Young's modulus as a function of the strain level.

TABLE 2: $R^{2}$ values of the linear model for TA and EDL specimens in the initial region of the stress-strain curve.

\begin{tabular}{lcccccc}
\hline Mouse & \multicolumn{1}{c}{ TA } & & \multicolumn{2}{c}{ EDL } \\
$R^{2}$ & $E_{\text {lin }}(\mathrm{kPa})$ & $\varepsilon_{\max }(\%)$ & $R^{2}$ & $E_{\text {lin }}(\mathrm{kPa})$ & 37.18 \\
2 & 0.9909 & 52.29 & 8.37 & 0.991 & 0.9954 & 36.84 \\
3 & 0.9897 & 56.73 & 7.05 & 0.9913 & 26.24 & 16.11 \\
4 & 0.9878 & 29.56 & 8.61 & 0.9944 & 38.55 & 14.45 \\
5 & 0.9866 & 25.71 & 6.52 & 0.9898 & 24.07 & 11.14 \\
6 & 0.9813 & 29.29 & 10.39 & 0.9855 & 37.89 & 14.97 \\
Average & - & - & - & & $33.46 \pm 6.50$ & 10.28 \\
\hline
\end{tabular}

${ }^{* *} p<0.01$ versus EDL.

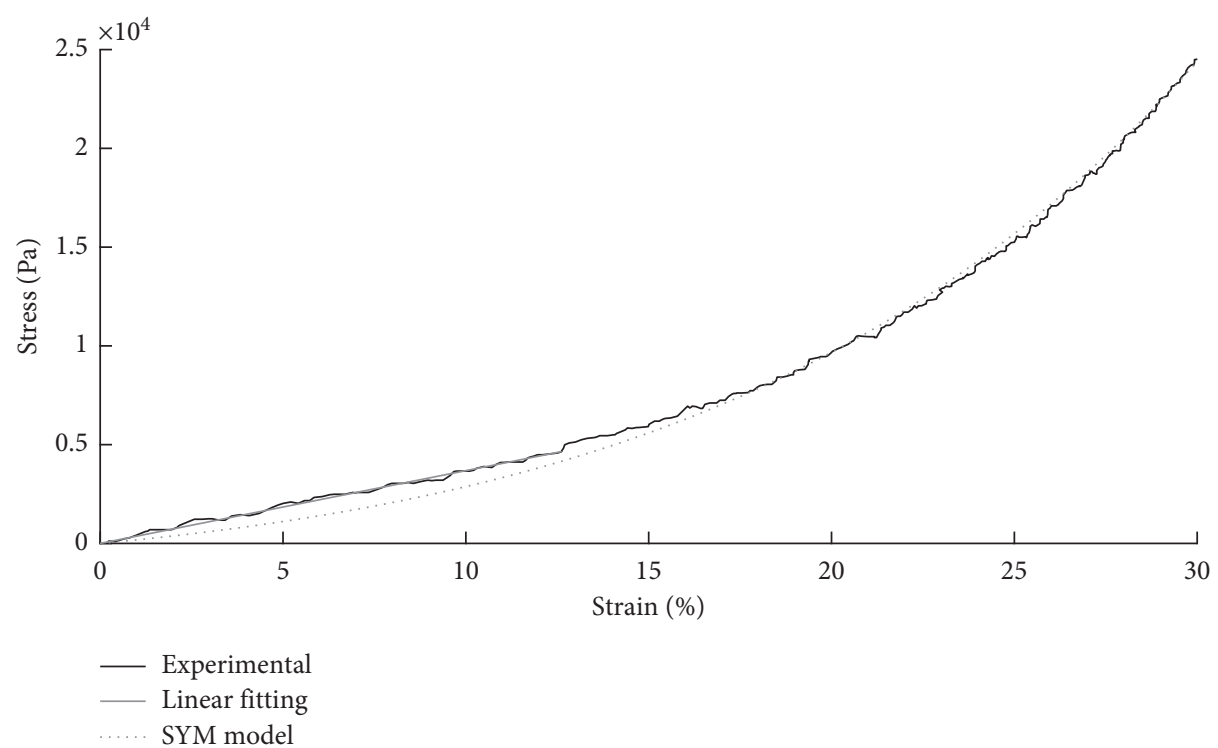

FIGURE 4: Example of fitting curves (dotted grey: SYM model; solid grey: linear model) and experimental data (black) for an EDL specimen.

and with the SYM model, respectively. Table 3 shows the results obtained for all the specimens, and Figure 5 shows an example of the results for one EDL muscle: for all the tested specimens, except for the initial and the final portion of the analyzed curve, in which the residuals are very similar for the two tested models, in the central part of the curve, the linear model better describes the experimental data. As a result of this, the average value of the linear model's residuals was lower than the average value of the SYM model's residual for all the tested specimens. When comparing the residual distributions, a statistically significant difference was found for all the tested specimens except that for one TA (Table 3), with the residuals computed for the linear model being extremely lower than residuals computed for the SYM model. 
TABLE 3: Residuals $(\mathrm{Pa})$ of the linear model and the SYM model in the initial linear region of the stress-strain curve.

\begin{tabular}{|c|c|c|c|c|}
\hline \multirow{2}{*}{ Mouse } & \multicolumn{2}{|c|}{ TA } & \multicolumn{2}{|c|}{ EDL } \\
\hline & Linear model & SYM model & Linear model & SYM model \\
\hline 1 & $148.52 \pm 87.21$ & $243.35 \pm 186.80^{* *}$ & $235.71 \pm 122.02$ & $323.63 \pm 194.28^{* *}$ \\
\hline 2 & $127.71 \pm 71.76$ & $182.12 \pm 102.46^{* * *}$ & $137.22 \pm 86.71$ & $437.38 \pm 299.13^{* * * *}$ \\
\hline 3 & $134.46 \pm 79.46$ & $194.83 \pm 150.90$ & $136.97 \pm 90.39$ & $558.88 \pm 334.03^{* * * *}$ \\
\hline 4 & $60.77 \pm 49.94$ & $264.03 \pm 162.52^{* * * *}$ & $137.65 \pm 104.61$ & $287.49 \pm 221.99^{* * * *}$ \\
\hline 5 & $120.23 \pm 74.62$ & $448.04 \pm 250.97^{* * * *}$ & $99.56 \pm 80.81$ & $440.18 \pm 313.01^{* * * *}$ \\
\hline 6 & - & - & $149.56 \pm 105.60$ & $328.61 \pm 189.40^{* * * *}$ \\
\hline
\end{tabular}

Average data are expressed as mean $\pm \mathrm{SD} .{ }^{* *} p<0.01,{ }^{* * *} p<0.001$, and ${ }^{* * * *} p<0.0001$ versus the linear model.

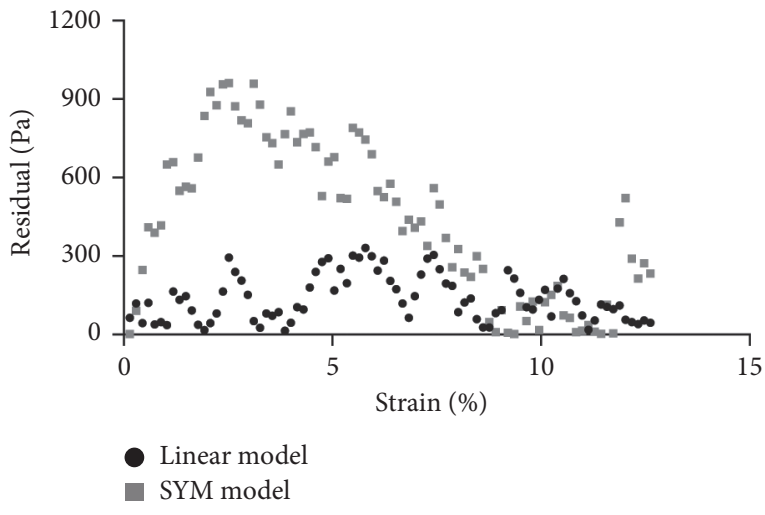

(a)

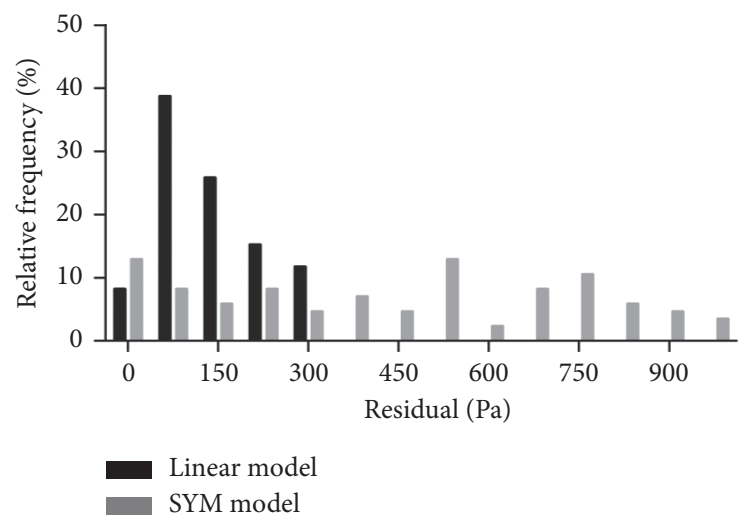

(b)

Figure 5: Example of a residual analysis for an EDL specimen ( $n: 2$ in Table 2) computed in the initial linear region for the two applied models (a) and their frequency distribution (b).

\section{Conclusions}

The aim of this study was to characterize, ex vivo, the mechanical response of two different types of mice muscles to an external force, the Tibialis Anterior (TA) and the Extensor Digitorum Longus (EDL), by introducing the nonlinearity in the general theory of linear elasticity. Elastography techniques are based on Hooke's law by assuming that soft tissues act as linear elastic materials; however, under large deformations, they exhibit a nonlinear elastic behavior, and a proper understanding of their mechanical properties is necessary to avoid errors in the elastogram and in the interpretation of the results. Indeed, the possibility of a more accurate identification of tissues' mechanical properties results indeed crucially in elastography approach. The comparison between the average strain values undergone by TA and EDL muscles showed that they exhibit distinct strain hardening effects, with the EDL being more compliant. The use of the SYM model already employed for the porcine hearth aortic valve [35] and to assess the passive behavior of skeletal muscle in compression $[33,36]$ provided a very good fit to the experimental data. The SYM model allowed to characterize how fast the stiffness of each specimen was raised with the increase of the applied load outside the linear elastic region and to predict Young's modulus for large deformations. Because of a more compliant behavior of EDL muscles, the maximum value of Young's modulus obtained through the SYM model resulted to be significantly higher in
TA muscles. However, the SYM model did not allow getting a single $E$ value to be associated with each muscle type. Due to the high variability of the strain as a function of the stress under large deformations, in strain elastography, reasonable strain signal-to-noise ratio and strain contrast between these two tissues may not be reached, and, in shear wave elastography, they may be measured having the same elasticity. The use of a recursive technique allowed obtaining an assessment of the part of the region in which the tissue linear response is more prevalent on the nonlinear one. Interestingly, even if the SYM model provided a good fitting of the entire $\sigma-\varepsilon$ relationship, in the initial part of the curve, the linear model provided an even more accurate modelling. This linear region was found to reach a higher strain value, on average, for EDL muscles than for TA ones, in accordance with the more compliant behavior previously measured for EDL specimens. Once the linear region was identified, it was possible to associate a unique value of Young's modulus to each muscle type, reducing the likelihoods of misinterpretation of the elastography outcomes. Again, it has to be remarked that the high variability obtained in the estimated linear modulus even for samples that are excised from very similar and controlled animal confirms the need for assessing the exact elasticity for each sample of interest. Of note, in this study, the tissue viscosity was considered negligible, but biological tissues, being viscoelastic, exhibit a stress dependence on the rate of straining. Anyway, our specimens were tested at a very slow rate to perform 
quasistatic indentations, minimizing the effect of viscoelasticity. As expected, the strain as a function of the stress exhibits a high variability; therefore results from this experimental study encourage the adoption of the models employed here to predict both the nonlinear and linear elastic responses of compressed soft tissues to provide, together with the measurement of the force applied during elastography techniques, a real-time correction of the measurements to avoid misinterpretation of the results. Finally, an improved version of the model proposed here may be therefore developed to consider the small contribution of viscosity, which can also contribute to reducing the variability obtained for the linearized values of $E$.

\section{Data Availability}

The data used to support the findings of this study are included within the article. Specific requests may be forwarded to the corresponding author.

\section{Conflicts of Interest}

The authors declare that they have no conflicts of interest.

\section{Authors' Contributions}

E. Rizzuto and R. De Luca contributed equally to this work.

\section{References}

[1] J. Ophir, "Elastography: imaging the elastic properties of soft tissues with ultrasound," Journal of Medical Ultrasonics, vol. 29, no. 4, pp. 155-171, 2002.

[2] J. Bamber, D. Cosgrove, C. F. Dietrich et al., "EFSUMB guidelines and recommendations on the clinical use of ultrasound elastography. part 1: basic principles and technology," Ultraschall in der Medizin, vol. 34, no. 2, pp. 169-184, 2013.

[3] C. Dietrich, J. Bamber, A. Berzigotti et al., "EFSUMB guidelines and recommendations on the clinical use of liver ultrasound elastography, update 2017 (long version)," Ultraschall der Medizin - Europe Journal Ultrasound, vol. 38, no. 4, pp. e16-e47, 2017.

[4] R. G. Barr, "Shear wave imaging of the breast: still on the learning curve," Journal of Ultrasound in Medicine, vol. 31, no. 3, pp. 347-350, 2012.

[5] R. G. Barr, "Sonographic breast elastography: a primer," Journal of Ultrasound in Medicine, vol. 31, no. 5, pp. 773-783, 2012.

[6] T. A. Krouskop, T. M. Wheeler, F. Kallel, B. S. Garra, and T. Hall, "Elastic moduli of breast and prostate tissues under compression," Ultrasonic Imaging, vol. 20, pp. 260-274, 1998.

[7] A. P. Sarvazyan, O. V Rudenko, S. D. Swanson, J. B. Fowlkes, and S. Y. Emelianov, "Shear wave elasticity imaging: a new ultrasonic technology of medical diagnostics," Ultrasound in Medicine and Biology, vol. 24, no. 9, pp. 1419-1435, 1998.

[8] G. Ferraioli, C. Filice, L. Castera et al., "WFUMB guidelines and recommendations for clinical use of ultrasound elastography: part 3: liver," Ultrasound in Medicine and Biology, vol. 41, no. 5, pp. 1161-1179, 2015.

[9] D. Cosgrove, F. Piscaglia, J. Bamber et al., "EFSUMB guidelines and recommendations on the clinical use of ultrasound elastography. Part 2: clinical applications," Ultraschall in der Medizin, vol. 34, no. 3, pp. 238-253, 2013.

[10] A. A. Oraevsky, B. Clingman, J. Zalev, A. T. Stavros, W. T. Yang, and J. R. Parikh, "Clinical optoacoustic imaging combined with ultrasound for coregistered functional and anatomical mapping of breast tumors," Photoacoustics, vol. 12, pp. 30-45, 2018.

[11] G. Y. Li and Y. Cao, "Mechanics of ultrasound elastography," Proceedings of the Royal Society A: Mathematical, Physical and Engineering Sciences, vol. 473, no. 2199, 2017.

[12] C. Bastard, Y. Mofid, J. Oudry, J.-P. Remenieras, V. Miette, and L. Sandrin, "Assessment of the elastic properties of heterogeneous tissues using transient elastography: application to the liver," in Proceedings of the 2008 IEEE Ultrasonics Symposium, pp. 317-320, Beijing, China, November 2008.

[13] J.-L. Gennisson, T. Deffieux, M. Fink, and M. Tanter, "Ultrasound elastography: principles and techniques," Diagnosis Intervention Imaging, vol. 94, no. 5, pp. 487-495, 2013.

[14] L. Mulazzani, L. Mulazzani, V. Salvatore et al., "Point shear wave ultrasound elastography with Esaote compared to realtime $2 \mathrm{D}$ shear wave elastography with supersonic imagine for the quantification of liver stiffness," Journal of Ultrasound, vol. 20, no. 3, pp. 213-225, 2017.

[15] M. Tanter, M. Pernot, G. Montaldo et al., "Real time quantitative elastography using supersonic shear wave imaging," in Proceedings of the 2010 IEEE International Symposium on Biomedical Imaging: From Nano to Macro, pp. 276-279, Rotterdam, Netherlands, April 2010.

[16] N. Šarabon, Ž. Kozinc, and N. Podrekar, "Using shear-wave elastography in skeletal muscle: a repeatability and reproducibility study on biceps femoris muscle," PLoS One, vol. 14, no. 8, Article ID e0222008, 2019.

[17] J. A. Ryu and W. K. Jeong, "Current status of musculoskeletal application of shear wave elastography," Ultrasonography, vol. 36, no. 3, pp. 185-197, 2017.

[18] A. Athanasiou, A. Tardivon, M. Tanter et al., "Breast lesions: quantitative elastography with supersonic shear imaging-preliminary results," Radiology, vol. 256, no. 1, pp. 297-303, 2010.

[19] W. A. Berg, D. O. Cosgrove, C. J. Doré et al., "Shear-wave elastography improves the specificity of breast US: the BE1 multinational study of 939 masses," Radiology, vol. 262, no. 2, pp. 435-449, 2012.

[20] P. Tortoli, T. Morganti, G. Bambi, C. Palombo, and K. V. Ramnarine, "Noninvasive simultaneous assessment of wall shear rate and wall distension in carotid arteries," Ultrasound Medical Biology, vol. 32, no. 11, pp. 1661-1670, 2006.

[21] Y. Xiao, J. Zeng, M. Qian, R. Zheng, and H. Zheng, "Quantitative analysis of peri-tumor tissue elasticity based on shear-wave elastography for breast tumor classification," in Proceedings of the 2013 35th Annual International Conference of the IEEE Engineering in Medicine and Biology Society (EMBC), pp. 1128-1131, Osaka, Japan, September 2013.

[22] D. O. Cosgrov, W. A. Berg, C. J. Doré et al., "Shear wave elastography for breast masses is highly reproducible," European Radiology, vol. 22, no. 5, pp. 1023-1032, 2012.

[23] F. Hug, K. Tucker, J.-L. Gennisson, M. Tanter, and A. Nordez, "Elastography for muscle biomechanics: toward the estimation of individual muscle force," Exercise and Sport Sciences Reviews, vol. 43, no. 3, pp. 125-133, 2015.

[24] M. Bird, D. Le, J. Shah et al., "Characterization of local muscle fiber anisotropy using shear wave elastography in patients with chronic myofascial pain," in Proceedings of the 2017 IEEE 
International Ultrasonics Symposium (IUS), p. 1, Washington, DC, USA, September 2017.

[25] C. Dorado Cortez, L. Hermitte, A. Ramain, C. Mesmann, T. Lefort, and J. B. Pialat, "Ultrasound shear wave velocity in skeletal muscle: a reproducibility study," Diagnosis Intervention Imaging, vol. 97, no. 1, pp. 71-79, 2016.

[26] E. E. Drakonaki, G. M. Allen, and D. J. Wilson, "Ultrasound elastography for musculoskeletal applications," British Journal of Radiology, vol. 85, no. 1019, pp. 1435-1445, 2012.

[27] B. R. Chintada, R. Rau, and O. Goksel, "Nonlinear characterization of tissue viscoelasticity with acoustoelastic attenuation of shear-waves," 2020, https://arxiv.org/abs/2002. 12908.

[28] Y. Qiu, F. R. Zaki, N. Chandra, S. A. Chester, and X. Liu, "Nonlinear characterization of elasticity using quantitative optical coherence elastography," Biomedical Optics Express, vol. 7, no. 11, p. 4702, 2016.

[29] R. G. Barr and Z. Zhang, "Effects of precompression on elasticity imaging of the breast: development of a clinically useful semiquantitative method of precompression assessment," Journal of Ultrasound in Medicine, vol. 31, no. 6, pp. 895-902, 2012.

[30] T. Varghese, J. Ophir, and T. Krouskop, "Nonlinear stressstrain relationships in tissue and their effect on the contrastto-noise ratio in elastograms," Ultrasound Medical Biology, vol. 26, no. 5, pp. 839-851, 2000.

[31] B. C. W. Kot, Z. J. Zhang, A. W. C. Lee, V. Y. F. Leung, and S. N. Fu, "Elastic modulus of muscle and tendon with shear wave ultrasound elastography: variations with different technical settings," PLoS One, vol. 7, no. 8, Article ID e44348, 2012.

[32] C. T. McKee, J. A. Last, P. Russell, and C. J. Murphy, "Indentation versus tensile measurements of Young's modulus for soft biological tissues," Tissue Engineering Part B Reviews, vol. 17, no. 3, pp. 155-164, 2011.

[33] M. Van Loocke, C. G. Lyons, and C. K. Simms, "A validated model of passive muscle in compression," Journal of Biomechanics, vol. 39, no. 16, pp. 2999-3009, 2006.

[34] B. Calvo, A. Ramírez, A. Alonso et al., "Passive nonlinear elastic behaviour of skeletal muscle: experimental results and model formulation," Journal of Biomechanics, vol. 43, no. 2 , pp. 318-325, 2010.

[35] J. Li, X. Y. Luo, and Z. B. Kuang, "A nonlinear anisotropic model for porcine aortic heart valves," Journal of Biomechanics, vol. 34, no. 10, pp. 1279-1289, 2001.

[36] M. Van Loocke, C. G. Lyons, and C. K. Simms, "Viscoelastic properties of passive skeletal muscle in compression: stressrelaxation behaviour and constitutive modelling," Journal of Biomechanics, vol. 41, no. 7, pp. 1555-1566, 2008.

[37] R. De Luca, E. Rizzuto, and Z. Del Prete, "Characterizing the non-linear elastic properties of ex-vivo mouse tissues," in Proceedings of the 2016 IEEE International Symposium on Medical Measurements and Applications (MeMeA), pp. 1-4, Benevento, Italy, May 2016. 